Caballero-García, P.A. \& Sánchez-Ruíz, S. (2018). La felicidad en estudiantes universitarios. ¿Existen diferencias según género, edad o elección de estudios? Revista Electrónica Interuniversitaria de Formación del Profesorado, 21(3), 1-18.

\title{
La felicidad en estudiantes universitarios. ¿Existen diferencias según género, edad o elección de estudios? *
}

\author{
Presentación A. Caballero-García, Sara Sánchez Ruiz \\ Universidad Camilo José Cela, Madrid, España
}

\section{Resumen}

El trabajo que presentamos estudia la felicidad en estudiantes universitarios, y las posibles diferencias por género, edad, y elección de estudios que pudieran surgir en su percepción subjetiva, derivadas de una intervención en el aula basada en emociones positivas y creatividad. Utilizamos un diseño cuasi-experimental con pretest y postest. La muestra estuvo formada por 83 estudiantes universitarios y de Grados Técnicos Superiores, que fueron evaluados con la escala de Felicidad Subjetiva de Lyubomirsky y Lepper (1999) y el Cuestionario de Felicidad de Oxford de Hills y Argyle (2002). Los resultados muestran que no existen diferencias significativas en la percepción de felicidad por género, edad y elección de estudios, sin embargo, el programa de intervención en emociones positivas fue efectivo y aumentó significativamente la felicidad subjetiva de los alumnos del grupo experimental frente al control. La felicidad ha sido asociada positivamente al éxito académico, favorece el afrontamiento de situaciones estresantes de la vida universitaria; tiene efectos positivos en la salud y el bienestar personal y social, contribuye a la calidad de vida. Dada la importancia de preparar al alumno para la vida laboral y los beneficios que las emociones positivas les aportan defendemos su uso integrado en las aulas universitarias.

\section{Palabras clave}

Felicidad; género; edad; elección de estudios.

\section{Contacto:}

Presentación A. Caballero García. Facultad de Educación. Universidad Camilo José Cela. C/ Castillo de Alarcón, 49 • Urb. Villafranca del Castillo · 28692 Madrid. Email: pcaballero@ucjc.edu

\footnotetext{
* Trabajo desarrollado por el GdI ASE, dentro del Proyecto I+D titulado HACKER \& HAPPY. Originales, Audaces e inteligentes, $\mathrm{N}^{\circ}$ expediente: 2015-22, financiado por la Universidad Camilo José Cela, en su IV Convocatoria de Ayudas a la Investigación
} 


\title{
Happiness in university students. Are there differences depending on gender, age, or choice of studies?
}

\begin{abstract}
The present work deals with the study of happiness on university students, and the possible differences depending on their gender, age and choice of studies that may arise in their subjective perception, derived from an intervention in the classroom based on positive emotions and creativity. We use a quasi-experimental design with pretest and posttest. The sample consisted in 83 university students and Higher Technical Degrees, who were evaluated with the "Subjective Happiness scale of Lyubomirsky and Lepper" (1999) and the "Oxford Happiness Questionnaire of Hills and Argyle" (2002). The results show that there are no significant differences in the perception of happiness depending on gender, age and choice of studies, however, the intervention in positive emotions program was effective and the subjective happiness of the students in the experimental group increased significantly compared to the control group. Happiness has been positively associated to academic success; encourages coping with stressful situations of university life; has positive effects on health and on personal and social well-being, also contributes improving people quality of life. Given the importance of preparing the student for working life and the benefits that positive emotions provide, we support their integrated use in university classrooms.
\end{abstract}

\section{Key words}

Happiness; sex; age; choice studies.

\section{Introducción}

Si algo ha marcado los estudios en el EEES ha sido el diseño de una formación basada en competencias. Las competencias determinan los saberes y actitudes correspondientes a las funciones profesionales de los titulados (Cabrerizo, Rubio y Castillejo, 2007). En este sentido, la competencia de "aprender a aprender" ha cobrado mayor importancia que nunca, al tiempo que han empezado a entrar en juego, en el panorama educativo universitario, otras variables vinculadas con la inteligencia emocional y la Psicología positiva.

La vida universitaria está compuesta por un gran número de factores estresantes, tales como, realización de exámenes, trabajos individuales y grupales, prácticas, fechas de entrega, establecimiento de relaciones sociales, etc. (Figueiredo Ferraz, Cardona, y GilMonte, 2009), que pueden amenazar el bienestar, tanto físico como psicológico, de los estudiantes (Cova Solar et al., 2007). Esto conlleva la necesidad de estrategias que les permita afrontarlos de manera adecuada (Salavera Borás y Usán Supervía, 2017).

En la última década, se han incrementado los estudios sobre el bienestar subjetivo, que como constructo incluye dos componentes claramente diferenciados y que han seguido líneas de investigación paralelas (Veloso-Besio, Cuadra-Peralta, Antezana-Saguez, Avendaño-Robledo y Fuentes-Soto, 2013): por un lado, los juicios cognitivos sobre satisfacción con la vida y, por otro, las evaluaciones afectivas sobre el humor y las emociones, equivalentes a la felicidad subjetiva (Padrós, Martínez, Gutiérrez-Hernández y Medina, 2010). El componente emocional, alusivo a la felicidad subjetiva, corresponde a la 
preponderancia de los sentimientos o afectos positivos sobre los negativos, (García, 2002), y es el que ha sido objeto de nuestra atención en este estudio.

La felicidad siempre ha tenido, para hombres y mujeres, singular valor entre las metas de vida (Alarcón, 2003). Algunos autores como Diener (2000) han afirmado incluso que la felicidad es mejor indicador de la calidad de vida que el bienestar o la salud. La literatura indica que las personas felices son exitosas en múltiples dominios de la vida, tales como matrimonio, amistad, trabajo, rendimiento, sueldo y salud (Lyubomirsky, King y Diener, 2005; Ramírez y Fuentes, 2013; Scorsolini-Comin y Dos Santos, 2012).

Cuando se ha estudiado en el ámbito universitario, se ha comprobado que los estudiantes felices tienen más probabilidades de finalizar sus estudios universitarios (Frisch et al., 2005; González, Castro y Martín, 2011; Wintre et al., 2011). La felicidad, ha sido asociada positivamente con el éxito académico (Heikkila, Lonka, Niemen y Niemivirita, 2012; Lyubomirsky, et al., 2005; Scorsolini-Comin y Dos Santos, 2012; Oliver, 2000) y algunos autores (Wright y Cropanzano, 2000) han afirmado incluso que es el mejor predictor del rendimiento. En esta línea, Medrano (2014) sostiene que la felicidad está en la base del cumplimiento de metas, del planteamiento de otras nuevas, como también guarda relación con la satisfacción de vida y la mejora de las expectativas personales entre otros aspectos.

Las personas felices tienen mejores trabajos (con mayor autonomía, significado y variedad de tareas) (Staw, Sutton, y Pelled, 1994), están más satisfechas con estos (Connolly y Viswesvaran, 2000), tienen más iniciativa y curiosidad para explorar nuevas opciones (Kashdan y Roberts, 2004), están más abiertas al cambio (Bilbao, Techio y Páez, 2007), pasan menos tiempo desempleadas (Diener, Nickerson, Lucas y Sandvik, 2002), obtienen mejores calificaciones de sus supervisores (Staw, Sutton y Pelled, 1994) y reciben mejores puntajes en calidad en el trabajo, productividad y creatividad (Cropanzano y Wright, 1999; Wright y Staw, 1999), por lo que ocupan más puestos de supervisión ( Graen, 1976) y reciben mayores ingresos (Diener y Biswas-Diener, 2002) que otros trabajadores.

De igual manera se ha demostrado que los afectos positivos aumentan la resiliencia, la capacidad de superar situaciones adversas (Fredrickson y Joiner, 2002) y estresantes, y de desarrollarse profesionalmente (Ong, Bergeman, Bisconti y Wallace, 2006). Mejoran la capacidad de atención, producen una expansión y flexibilidad cognitiva (Fredrickson y Branigan, 2005), y aumentan la intuición (Bolte, Goschke y Kuhl, 2003) y la creatividad (Isen, Daubman y Nowicki, 1987) de las personas; pudiéndose afirmar que felicidad y bienestar suelen asociarse con la salud física, mental y la creatividad, siendo factores protectores de cuestiones tan relevantes como la depresión y el suicidio (Moyano y Ramos, 2007).

Pero la capacidad de ser más o menos felices no es algo que nos venga dado, la construimos a lo largo de la vida con las elecciones que hacemos (Headey, 2010). En este sentido, podemos afirmar que la felicidad puede enseñarse y aprenderse. Educar en felicidad significa educar en salud y bienestar, condiciones para superar algunas dificultades de aprendizaje y adquirir conocimiento significativo (Caballero-García, Carretero Cenjor y Fernández Palop, 2015; Caballero-García, Ruano y Sánchez, 2018). Teniendo en cuenta los beneficios que nos aporta y su contribución al desarrollo personal, académico, social y profesional, una educación orientada a la felicidad se justifica como necesaria para preparar a nuestros estudiantes para el futuro profesional. Dada la importancia de preparar al alumno para la vida laboral, resulta fundamental trabajar su felicidad desde la universidad. "Una educación universitaria debería ayudar a lograr la felicidad del alumno y, con ella, la sociedad en su conjunto" (González, 2014, p.67).

Respecto a cómo se comporta la felicidad por género, no puede afirmarse que haya resultados concluyentes. Hay autores (como Mookherjee, 1997; Velásquez et al., 2008; 
Wood, Rhodes y Whelan, 1989) que han encontrado que las mujeres muestran mayores niveles de felicidad que los hombres; mientras que otros autores (como Alarcón, 2001; Argyle, 1990; Diener y Diswas, 2008; Laca, Verdugo y Guzmán, 2005; Seligman, 2011; Salavera Borás y Usán Supervía, 2017; Torres-Pinazo et al., 2017 entre otros) no han hallado diferencias significativas al comparar las medias aritméticas obtenidas por varones y mujeres en felicidad y satisfacción con la vida.

Cuando se ha evaluado con la escala de Felicidad Subjetiva de Lyubomirsky y Lepper (1999) en muestras de estudiantes universitarios, se han encontrado diferencias significativas por género. Las mujeres realizan más conductas para procurarse felicidad (Torres Deik, Moyano-Díaz y Páez, 2014) y parecen mostrar mayor felicidad, bienestar o satisfacción con la vida que los hombres (Laca et al., 2005; Muratori, 2015; Quevedo, Villalobos y Abella, 2014; Vera-Noriega y Rodríguez, 2007).

Los mismos resultados se logran cuando la felicidad se ha medido con otros instrumentos, como las escalas de Ryff (1989) y su adaptación española (Díaz et al., 2006) o la Escala de Felicidad de Lima (EFL), desarrollada por Alarcón (2006) (Castilla, Caycho y Ventura-León, 2016; Muratori, 2015; Toribio y López, 2012). Lo cual concuerda con estudios previos (Francis, 1999; Lu, 1996; Mookherjee, 1997; Seligman, 2011; Wood, Rhodes et al, 1989).

Las mujeres también muestran mejores niveles que los hombres en dominio del entorno y propósito en la vida, bienestar psicológico y contribución del bienestar social (Muratori et al., 2012; Zubieta, Beramendi, Sosa y Torres, 2010; Zubieta y Delfino, 2010).

Cuando se ha estudiado la relación entre edad y felicidad, no se ha encontrado una asociación lineal ascendente entre ambas variables; sin embargo, los estudios demuestran que la felicidad y la satisfacción con la vida no declina con la edad (Alarcón, 2001; Diener y Suh, 1998). Contrariamente a lo que se pensaba, la curva del bienestar no tiene una forma de $U$, con el máximo de la curva entre los jóvenes y con el mínimo situado en la mediana edad, entre treinta y cuarenta y cinco años, cuando las responsabilidades familiares y laborales son mayores (Oswald, 1997), sino que las personas se vuelven más felices con los años (Ahn, Mochón Morcillo y Juan, 2012; Helliwell y Putnam, 2004). Las puntuaciones medias más altas de felicidad fueron obtenidas por sujetos de 50 y 60 años y, a partir de la década de los setenta, empieza a decaer (Frijters y Beatton, 2012; Mroczek y Kolarz, 1998; Mroczek y Spiro, 2005). Estos resultados son importantes toda vez que, muy a menudo, se ha supuesto todo lo contrario, que las personas mayores son las menos felices, cuando la evidencia empírica parece demostrar que no es así (Alarcón, 2011).

La evidencia empírica demuestra que las personas de mayor edad presentan mayores niveles de autonomía y dominio del entorno, otorgando un mayor sentido a sus experiencias y teniendo una mayor capacidad para controlar el contexto social a fin de satisfacer sus necesidades y valores, en comparación con los más jóvenes (Fernández et al., 2013; Muratori, 2015; Ryff, 1989; Zubieta, Muratori y Mele, 2012). A mayor edad se adquiere una mayor seguridad y experiencia en las tareas vitales y una menor vulnerabilidad al estrés o tensión emocional reactiva a los sucesos vitales. Las expresiones de conductas positivas contribuyen a mejorar las relaciones personales, además de servir como factor de protección ante diversos problemas (Romero, 2015). En general, la edad provee al individuo de más recursos y habilidades personales a la hora de comunicarse y actuar con otras personas, facilitando la resolución de problemas (García Mazzieri, 2012). A mayor edad, las personas se sienten más valoradas socialmente, y esto permite orientar los pensamientos hacia situaciones de carácter más positivo (Moyano y Ramos, 2007).

Los afectos positivos guardan una estrecha correlación con la felicidad (Alarcón, 2001; Carstensen, 1991, 1995; Labovic-Vief y Blanchard-Fields, 1982). Los adultos mayores regulan 
mejor sus emociones, y aprenden a minimizar los efectos negativos y a maximizar los positivos. Los jóvenes tienden a disfrutar en menor medida el presente que las personas mayores.

En cuanto a la felicidad como valor, la revisión bibliográfica realizada nos ha llevado a la conclusión de que la familia como la felicidad, son los primeros valores por antonomasia dentro de la jerarquía de los estudiantes universitarios (Angelucci et al., 2008). Encontramos, además, que los alumnos de Ciencias de la Educación suelen mostrar una clara inclinación hacia los valores emocionales (De la Torre y Tejada, 2007) frente a otros estudios. Esto suscitó en nosotros la curiosidad por conocer si sucedía lo mismo con la felicidad en ésta y otras carreras universitarias. En nuestros análisis de los estudios sobre felicidad que se han desarrollado con muestras universitarias, apenas encontramos casos que hayan tenido en cuenta la influencia que en ella pueda tener la titulación de procedencia, y la mayoría de ellos no encuentran diferencias significativas entre las distintas carreras.

En esta línea, Velásquez (2008) utilizó una amplia muestra con estudiantes de diferentes áreas del conocimiento: Matemáticas, Ingeniería, Medicina, Derecho, Educación y Administración de empresas, y llegó a la conclusión de que no existían diferencias significativas en el bienestar psicológico entre ellas. Galiana (2015), por su parte, midió la felicidad con una amplia muestra universitaria, diferenciando varios ámbitos: Ingeniería, Salud, Ciencias Sociales, Ciencias y Humanidades. Sus resultados tampoco encontraron diferencias significativas entre los titulados de las diferentes ramas del conocimiento. A la misma conclusión llegaron Prieto y Muñoz (2015) con una muestra universitaria de estudiantes de Derecho, Administración de Empresas e Ingeniería Informática, tras medir la felicidad mediante la EFL.

El trabajo que presentamos tiene antecedentes en estudios previos sobre el aprendizaje social y emocional (Caballero-García et al., 2015; Caballero-García, Carretero Cenjor y Sánchez, 2016; Caballero-García et al., 2018), y estudia la felicidad en estudiantes universitarios y las posibles diferencias que pudieran surgir en ella derivadas de una intervención basada en emociones positivas y creatividad, género, edad, y elección de estudios.

Sus objetivos específicos son: a) estudiar la homogeneidad existente, en un primer momento (pretest), entre el grupo control y el grupo experimental, respecto a la felicidad de los estudiantes; b) contrastar las posibles diferencias estadísticamente significativas, después del programa de intervención (postest), entre el grupo control y el grupo experimental, respecto a la felicidad de los estudiantes; c) analizar las posibles diferencias estadísticamente significativas en el pretest, en función del género, la edad y el ámbito educativo al que pertenecen los estudiantes; d) identificar las posibles diferencias estadísticamente significativas en el postest, en función del género, la edad y el ámbito educativo al que pertenecen los estudiantes.

Se enmarca dentro de los programas de Psicología Positiva que defienden la felicidad como facilitador del desempeño y la productividad de las personas en el contexto educativo, y programas que defienden el desarrollo de una cultura emocionalmente inteligente en la universidad y eligen la felicidad como un elemento potenciador del bienestar personal $y$ social y la empleabilidad de las personas.

\section{Método}

Utilizamos un diseño cuasi-experimental, con un grupo de control no equivalente (Cook y 
Campbell, 1979, 1986).

\section{Población y Muestra}

De la población universitaria y ciclos formativos de Educación Superior de la Comunidad de Madrid, en el estudio participaron alumnos procedentes de diferentes titulaciones de educación (Grados en Maestro en Educación Infantil y Educación Primaria) y Grados Superior de Técnico en Imagen para el Diagnóstico y Medicina Nuclear Dual, en Asesoría de Imagen Personal y Corporativa, Animación de Actividades Físicas y Deportivas, y Anatomía Patológica y Citodiagnóstico. La estrategia de muestreo fue no probabilística e intencional de voluntarios y aulas intactas. Estuvo compuesta por un total de 83 estudiantes, 59 (71.1\%) mujeres y $24(28.9 \%)$ hombres, con edades comprendidas entre 18 y 30 años. El grupo experimental estuvo compuesto por 40 estudiantes (48.2\%) y el grupo control por 43 estudiantes (51.8\%).

\section{Instrumentos}

Escala de Felicidad Subjetiva (Subjective Happiness Scale -SHS) de Lyubomirsky y Lepper (1999): es una medida global de felicidad percibida, que evalúa una categoría molar de bienestar como fenómeno psicológico global. Consta de 4 ítems con respuesta tipo Likert (de 1=muy en desacuerdo a 7=muy de acuerdo). Su corrección se hace mediante la sumatoria de los puntajes obtenidos y se dividen en el número total de ítem. Cumple los criterios psicométricos de confiabilidad, consistencia interna (entre 0,79 y 0,94 ), fiabilidad de re-test (entre 3 semanas y 1 año varió entre 0,55 y 0,90) y validez convergente (entre 0,52 y 0,72 con otras medidas de felicidad) y divergente con la escala de depresión de Beck (DBI) cuestionario de los cinco grandes $(\mathrm{BFI})$ y el cuestionario de optimismo disposicional (LOT-R), así como niveles altamente significativos, confiabilidad factorial entre 0,73 y 0,87 y estabilidad temporal de 0,61 , que confirman la idoneidad de su uso para medir el constructo de felicidad subjetiva (Hernández Moreno y Landero Hernández, 2014).

El Cuestionario de felicidad de Oxford (Oxford Happiness Questionnaire - OHQ) de Hills y Argyle (2002) fue creado con la intención de medir de forma global la felicidad personal. Está compuesto por 29 ítems que el sujeto debe valorar en una escala Likert con 6 posibles respuestas, desde "totalmente en desacuerdo" (1) a "totalmente de acuerdo" (6), de manera que las posibles puntuaciones totales presentan un rango de 29 a 174 puntos, correspondiendo las puntuaciones más altas a mayores niveles de bienestar subjetivo. La mitad de los ítems se plantean para que la respuesta se puntúe en orden inverso. Esta escala ha demostrado tener una alta consistencia interna. En su versión original presentó un alfa de Cronbach de 0,90 y una correlación test-retest a siete semanas de 0.78. La forma española del $\mathrm{OHQ}$ se desarrolló a partir de la escala original inglesa, mediante sucesivos procesos de traducción y retrotraducción. En general, puede considerarse que presenta características de fiabilidad y validez aceptables que justifican su uso en la evaluación del bienestar subjetivo en población española (Tomás-Sábado, 2014).

\section{Procedimiento de recogida de datos}

La recogida de datos se desarrolló en una sesión presencial de media hora de duración en la que se explicaron los objetivos de la investigación, los estudiantes aceptaron voluntariamente participar dentro del estudio y cumplimentaron los tests de felicidad en dos momentos del tiempo pretest y postest.

El plan de intervención se realizó únicamente en los grupos experimentales, entre las medidas pretest y postest, trabajando en el aula las emociones positivas, la creatividad, y la indagación de los alumnos de manera integrada dentro de las clases habituales y en 
diferentes materias de su carrera (Didáctica de la Matemática, Técnicas de Comunicación y Asesoramiento Educativo, El Currículum de la Educación Infantil, Observación e investigación en el aula, Educación Física, y Anatomía Patológica y Citodiagnóstico). La metodología desarrollada en las clases estuvo basada en emociones positivas y se desarrolló con técnicas Maker skills y Learning by doing, que se combinaron con el aprendizaje vivencial o experiencial (experiential learnig) y el aprendizaje basado en contexto (context-based learning). El profesor gestionó sus espacios y tiempos de aprendizaje desde el rol de formador, mentor, desarrollador y evaluador, manteniendo en todo momento el protagonismo del alumno y su aprendizaje. Los alumnos comprendieron la materia de un modo integral, en el que lo académico se combinó con lo práctico e investigativo (Robles y Caballero, 2010, 2011) y con lo humano y relacional. Trabajamos la curiosidad y las cualidades inherentes a la investigación como elementos básicos del bachillerato internacional, y aspectos clave para la transformación y el cambio, en la manera de aprender y enseñar en el aula y para conseguir mejoras en la práctica educativa.

El grupo control tuvo sus medidas de felicidad pretest y postest, en similares momentos que el grupo experimental, y desarrolló sus clases (El Currículum de la Educación Infantil, Observación e Investigación en el Aula, Imagen para el Diagnóstico y Medicina Nuclear y Asesoramiento de Imagen) con una metodología tradicional.

Una vez recogidos los datos se registraron, tabularon y prepararon para el tratamiento estadístico con SPSS (v. 24). Realizamos estadística descriptiva e inferencial no paramétrica, una vez realizadas las pruebas de normalidad y homocedasticidad pertinentes (Prueba $U$ de Mann Whitney, para comparaciones de medias de dos grupos y prueba de Kruskal Wallis para la comparación de más de dos grupos). El nivel de significación estadística fue de $a=, 05$.

\section{Resultados}

En relación al estudio del primer objetivo, referente a la homogeneidad existente, en el momento del pretest, entre el grupo control y el grupo experimental, respecto a la felicidad de los estudiantes, se pueden observar en la Tabla 1 las medias $(\bar{X})$ y desviaciones típicas $(\sigma)$ de las variables de la investigación.

Tal y como se aprecia, las puntuaciones de ambos test en un primer momento muestran, tanto en las valoraciones obtenidas del cuestionario de Oxford, como en la escala de Felicidad Subjetiva, que los alumnos pertenecientes al grupo experimental se manifiestan ligeramente más felices $\left(\overline{\mathrm{X}}_{\mathrm{OHQ \operatorname {exp }}}=4.45 ; \overline{\mathrm{X}}_{\mathrm{SHSexp}}=4.88\right)$ que los integrantes del grupo de control $\left(\bar{X}_{\mathrm{OHQC}}=4.40 ; \bar{X}_{\mathrm{SHSC}}=4.72\right)$, respectivamente. Pese a todo, la prueba $U$ de MannWhitney no arrojó diferencias significativas entre dichas opiniones, en ninguno de los casos $\left(p_{\mathrm{OHQ}}=.724 ; p_{\mathrm{SHS}}=.485\right)$.

Con respecto al segundo objetivo, relativo al contraste entre las posibles diferencias estadísticamente significativas en el postest, entre el grupo control y el grupo experimental, respecto a la felicidad de los estudiantes, tal y como se puede observar en la Tabla 2, de manera similar al caso anterior, las puntuaciones medias de ambos test arrojan valoraciones más elevadas en el caso de los alumnos pertenecientes al grupo experimental $\left(\bar{X}_{\text {OнQеxp }}=4.60 ; \bar{X}_{\text {SHSexp }}=5.05\right)$, frente al grupo de control $\left(\bar{X}_{\text {OHQc }}=4.27 ; \bar{X}_{\text {SHSC }}=4.56\right)$. Por tanto, estos estudiantes después del programa de intervención aseguran sentirse más felices, hallándose, además, diferencias estadísticamente significativas entre ambos grupos ( оно $_{\text {O }}=$ $\left..022 ; p_{\mathrm{SHS}}=.028\right)$. 
Tabla 1.

Estadísticos descriptivos y significación estadística de la Felicidad, en el pretest, según el grupo.

\begin{tabular}{ccccc}
\hline Pretest & & Grupo de control & Grupo experimental & Significación estadística \\
\hline \multirow{2}{*}{ OHQ } & $\bar{X}$ & 4.40 & 4.45 & .724 \\
\cline { 2 - 4 } & $\sigma$ & .591 & .636 & \\
\hline \multirow{2}{*}{ SHS } & $\bar{X}$ & 4.72 & 4.88 & .485 \\
\cline { 2 - 5 } & $\sigma$ & .935 & .999 & \\
\hline
\end{tabular}

Tabla 2.

Estadísticos descriptivos y significación estadística de la Felicidad, en el postest, según el grupo.

\begin{tabular}{ccccc}
\hline Postest & \multicolumn{1}{c}{ Grupo de control } & Grupo experimental & Significación estadística \\
\hline \multirow{2}{*}{ OHQ } & $\bar{X}$ & 4.27 & 4.60 & .022 \\
\cline { 2 - 4 } & $\sigma$ & .621 & .726 & \\
\hline \multirow{2}{*}{ SHS } & $\bar{X}$ & 4.56 & 5.05 & .028 \\
\cline { 2 - 4 } & $\sigma$ & .878 & .964 & \\
\hline
\end{tabular}

Dando respuesta al tercer objetivo, analizando ahora las posibles diferencias estadísticamente significativas en el pretest, en función del género, la edad y el ámbito educativo al que pertenecen los estudiantes, se presentan en la Tablas 3, 4 y 5 los diferentes resultados estadísticos para su estudio.

Concretamente, en la Tabla 3 se muestran las puntuaciones relativas a la felicidad de los alumnos, antes de la intervención, según sean estos mujeres $u$ hombres. Tal y como se observa, son las chicas, en ambos test, las que afirman sentirse más felices $\left(\bar{X}_{\mathrm{OHQm}}=4.52\right.$; $\left.\bar{X}_{S H S m}=4.88\right)$, que los chicos $\left(\bar{X}_{\mathrm{OHQh}}=4.18 ; \bar{X}_{\mathrm{SHSh}}=4.58\right)$, aunque no se encuentra significación estadística entre dichas diferencias encontradas ( $\left.p_{\mathrm{OHQ}}=.068 ; p_{\mathrm{SHS}}=.777\right)$.

Tabla 3.

Estadísticos descriptivos y significación estadística de la Felicidad, en el pretest, según el género.

\begin{tabular}{|c|c|c|c|c|}
\hline Pretest & & Mujeres & Hombres & Significación estadística \\
\hline \multirow{2}{*}{ OHQ } & $\bar{x}$ & 4.52 & 4.18 & \multirow{2}{*}{.068} \\
\hline & $\sigma$ & .506 & .776 & \\
\hline \multirow{2}{*}{ SHS } & $\bar{x}$ & 4.88 & 4.58 & \multirow{2}{*}{.777} \\
\hline & $\sigma$ & .731 & 1.377 & \\
\hline
\end{tabular}

A continuación, se analizan las puntuaciones pertenecientes a la felicidad de los discentes, antes de la intervención, según la edad de los mismos. De este modo, en esta Tabla 4 se 
aprecia como los alumnos más jóvenes, los que tienen menos de veinte años, en coherencia con las puntuaciones obtenidas en ambos test, son los que más felices aseguran sentirse $\left(\bar{X}_{\mathrm{OHQ}<20}=4.55 ; \bar{X}_{\mathrm{SHS}<20}=5.05\right)$. Pese a ello, no se encuentran diferencias estadísticamente significativas entre los tres grupos de edades de los estudiantes ( $\left.p_{\mathrm{OHQ}}=.260 ; p_{\mathrm{SHS}}=.846\right)$.

Tabla 4.

Estadísticos descriptivos y significación estadística de la Felicidad, en el pretest, según la edad del alumno.

\begin{tabular}{cccccc}
\hline Pretest & & Menos de 20 años & De 20 a 22 años & De 23 a 30 años & Significación estadística \\
\hline \multirow{2}{*}{ OHQ } & $\bar{X}$ & 4.55 & 4.38 & 4.42 & .260 \\
\cline { 2 - 5 } & $\sigma$ & .414 & .686 & .480 & \\
\hline \multirow{2}{*}{ SHS } & $\bar{X}$ & 5.05 & 4.75 & 4.50 & .846 \\
\cline { 2 - 5 } & $\sigma$ & .704 & 1.062 & .780 & \\
\hline
\end{tabular}

En cuanto a la felicidad del alumnado, según el nivel educativo de los mismos, la Tabla 5 muestra como el alumnado perteneciente al sector educación, según la escala de Felicidad Subjetiva y antes de la intervención realizada, se declara más feliz $\left(\bar{X}_{\text {SHSed }}=4.85\right)$ que los chicos que estudian titulaciones de carácter no educativo $\left(\bar{X}_{S H S x}=4.79\right)$, sin arrojar estas diferencias según el ámbito, significación estadística ( $\left.p_{\mathrm{OHQ}}=.841 ; p_{\mathrm{SHS}}=.794\right)$.

Tabla 5 .

Estadísticos descriptivos y significación estadística de la Felicidad, en el pretest, según el ámbito.

\begin{tabular}{ccccc}
\hline Pretest & & Ámbito educativo & Ámbito no educativo & Significación estadística \\
\hline \multirow{2}{*}{ OHQ } & $\bar{X}$ & 4.42 & 4.42 & .841 \\
\cline { 2 - 4 } & $\sigma$ & .680 & .606 & \\
\hline \multirow{2}{*}{ SHS } & $\bar{X}$ & 4.85 & 4.79 & .794 \\
\cline { 2 - 5 } & $\sigma$ & .801 & .989 & \\
\hline
\end{tabular}

Atendiendo, como último objetivo, a la identificación de las posibles diferencias estadísticamente significativas en el postest, en función del género, la edad y el ámbito educativo al que pertenecen los estudiantes, se presentan las Tablas 6, 7, y 8.

Concretamente en la Tabla 6 se exponen las puntuaciones de los resultados obtenidos según el género de los alumnos. Así pues, las alumnas continúan afirmando sentir mayor felicidad $\left(\bar{X}_{\mathrm{OHQm}}=4.52 ; \bar{X}_{\mathrm{SHSm}}=4.82\right)$ que los estudiantes varones $\left(\overline{\mathrm{X}}_{\mathrm{OHQh}}=4.29 ; \overline{\mathrm{X}}_{\mathrm{SHSh}}=4.81\right)$. De nuevo, no se hallan diferencias significativas ( $\left.p_{\mathrm{OHQ}}=.303 ; p_{\mathrm{SHS}}=.809\right)$.

En relación a las puntuaciones relativas a la felicidad de los discentes, después de la intervención, según la edad de los mismos, tal y como se observa en esta Tabla 7 , de nuevo el alumnado más joven es el que muestra un mayor índice de felicidad ( $\overline{\mathrm{X}}_{\mathrm{OHQ}<20}=4.54$; $\left.\overline{\mathrm{X}}_{\mathrm{SHS}<20}=4.91\right)$. Aun así, tampoco se encuentran diferencias estadísticamente significativas entre los tres grupos de edades de los estudiantes ( $\left.p_{\mathrm{OHQ}}=.613 ; p_{\mathrm{SHS}}=.763\right)$. 
Tabla 6.

Estadísticos descriptivos y significación estadística de la Felicidad, en el postest, según el género.

\begin{tabular}{ccccc}
\hline Postest & & Mujeres & Hombres & Significación estadística \\
\hline \multirow{2}{*}{ OHQ } & $\bar{X}$ & 4.52 & 4.29 & .303 \\
\cline { 2 - 4 } & $\sigma$ & .659 & .774 & .809 \\
\hline \multirow{2}{*}{ SHS } & $\bar{X}$ & 4.82 & 4.81 & \\
\cline { 2 - 4 } & $\sigma$ & .980 & .904 & \\
\hline
\end{tabular}

Tabla 7.

Estadísticos descriptivos y significación estadística de la Felicidad, en el postest, según la edad del alumno.

\begin{tabular}{cccccc}
\hline Postest & \multicolumn{1}{c}{ Menos de 20 años } & De 20 a 22 años & De 23 a 30 años & Significación estadística \\
\hline \multirow{2}{*}{ OHQ } & $\bar{X}$ & 4.54 & 4.37 & 4.58 & .613 \\
\cline { 2 - 5 } & $\sigma$ & .618 & .747 & .619 & \\
\hline \multirow{2}{*}{ SHS } & $\bar{X}$ & 4.91 & 4.76 & 4.90 & .763 \\
\cline { 2 - 5 } & $\sigma$ & 1.031 & .909 & 1.055 & \\
\hline
\end{tabular}

Analizando finalmente la felicidad del alumnado, según su nivel educativo, se muestran en la Tabla 8 que, según ambas escalas y después de la intervención realizada, el alumnado que cursa titulaciones en el contexto pedagógico, obtiene puntuaciones de felicidad más elevadas $\left(\bar{X}_{\text {SHSed }}=4.72 ; \bar{X}_{\text {SHSed }}=5.15\right)$ que los estudiantes que estudian titulaciones del sector no educativo $\left(\bar{X}_{\mathrm{SHSX}}=4.38 ; \overline{\mathrm{X}}_{\mathrm{SHSx}}=4,74\right)$, de nuevo sin arrojar estas diferencias según el ámbito, significación estadística ( $\left.p_{\mathrm{OHQ}}=.050 ; p_{\mathrm{SHS}}=.185\right)$.

Tabla 8.

Estadísticos descriptivos y significación estadística de la Felicidad, en el postest, según el ámbito.

\begin{tabular}{ccccc}
\hline Postest & & Ámbito educativo & Ámbito no educativo & Significación estadística \\
\hline \multirow{2}{*}{ OHQ } & $\bar{X}$ & 4.72 & 4.38 & .050 \\
\cline { 2 - 4 } & $\sigma$ & .699 & .684 & \\
\hline \multirow{2}{*}{ SHS } & $\bar{X}$ & 5.15 & 4.74 & .185 \\
\cline { 2 - 5 } & $\sigma$ & 1.068 & .914 & \\
\hline
\end{tabular}

\section{Discusión y conclusiones}

Algunas investigaciones han mostrado la importancia de las emociones positivas, no solo porque nos hacen sentir bien, sino también por la capacidad que tienen para brindar resultados exitosos en los distintos aspectos y áreas de nuestra vida, tanto individual, familiar, académico y laboral (Resino y Carrasco-Temiño, 2017). 
Partíamos de la necesidad de que nuestros estudiantes tuvieran estrategias para afrontar los desafíos de la vida universitaria y ayudas a su empleabilidad, y fomentábamos una educación emocional y positiva como propuesta de mejora y cambio en la universidad.

Todo plan de intervención necesita de evaluaciones previas que orienten el camino del cambio para la mejora. Con este fin quisimos conocer la felicidad de estudiantes universitarios de nuestro entorno en un estado inicial (curso 2017-2018) y estudiar las posibles diferencias que pudieran surgir en sus percepciones sobre ella derivadas de una intervención basada en emociones positivas y creatividad, género, edad, y elección de estudios, inserta en materias de su carrera.

Atendiendo al primer objetivo, referente a la homogeneidad existente, en el momento del pretest, respecto a la felicidad de los estudiantes (tanto en las valoraciones obtenidas del cuestionario de Oxford, como en la escala de Felicidad Subjetiva), los resultados obtenidos permiten concluir que no se encuentra diferenciación estadística entre las opiniones del alumnado perteneciente a los grupos control y experimental, por tanto, nuestro estudio ha partido de grupos uniformes.

En cambio, con respecto al segundo objetivo, en el momento del postest, sí que hayamos diferencias estadísticamente significativas entre las opiniones acerca de la felicidad de los grupos control y experimental, a favor de estos últimos. Los alumnos se sintieron más felices después de haber fomentado la felicidad en clase, con la intervención aplicada.

Dando respuesta al tercer y cuarto objetivos, en el momento inicial, no se observan diferencias significativas en función del género, la edad o el ámbito educativo de los alumnos. Tampoco encontramos diferencias significativas tras analizar las opiniones de los estudiantes en función del género, la edad y el ámbito educativo al que pertenecen, en el postest. Nuestros resultados se sitúan en línea con los trabajos de otros autores como Alarcón (2001), Argyle (1990), Diener y Diswas (2008), Laca, Verdugo et al. (2005), Seligman (2011), Salavera Borás y Usán Supervía (2017) y Torres-Pinazo et al. (2017), entre otros, que no hallaron diferencias significativas al comparar las medias aritméticas obtenidas por varones y mujeres en felicidad y satisfacción con la vida. Por tanto, el programa de intervención no arroja efectos distintos en función del género, edad o ámbito educativo del alumnado. Ha logrado fomentar la felicidad, a todos por igual, sin distinciones de género, ni de edad, ni de elección de estudios.

Se precisa mayor número de estudios diferenciales sobre el tratamiento de la felicidad en la universidad, en línea con los objetivos de nuestra investigación, que constaten con muestras más representativas la ligera tendencia encontrada hacia que las mujeres se perciban más felices que los hombres, en línea con los trabajos de otros autores como Laca et al. (2005), Mookherjee (1997), Muratori (2015), Quevedo et al. (2014), Vera-Noriega y Rodríguez (2007), Wood et al. (1989). En cuanto a la variable edad, los menores de 20 años de nuestra muestra se mostraron ligeramente más felices que los demás. Esto coincide con estudios (Ahn et al., 2012; Graham, 2005; Oswald, 1997) que concluyen que la felicidad tiene forma de $\mathrm{U}$, situándose los momentos de mayor felicidad en la juventud, descendiendo llegando a los 30 y volviendo a crecer en la madurez. Serían necesarios estudios con mayor intervalo de edad en los que puedan constatarse esta tendencia. Finalmente, haciendo alusión a la elección de estudios, observamos que los estudiantes de Grado de Maestro afirmaron sentirse ligeramente más felices que el resto de titulaciones estudiadas, coincidiendo con las investigaciones de De la Torre y Tejada (2007) donde los alumnos de Ciencias de la Educación muestran una clara inclinación hacia los valores emocionales frente a otros estudios. Los autores subrayan la importancia de desarrollar fortalezas positivas y habilidades socioemocionales en el contexto educativo (Fernández Berrocal y Extremera, 
2009). Se necesitan programas de intervención en la Universidad que, en línea con nuestros planteamientos, aseguren igualdad de oportunidades de los estudiantes, en cuanto al desarrollo de sus emociones y su felicidad subjetiva, teniendo en cuenta los beneficios que ésta reporta para el desarrollo personal, académico, social y laboral. Palomero Pescador (2003) subraya la necesidad de que se produzcan cambios importantes en la Universidad y en su profesorado, en aras de su doble misión pedagógica: formar profesionales competentes, a la vez que ciudadanos libres, ilustrados, respetuosos, democráticos, críticos, tolerantes y felices.

\section{Referencias}

Ahn, N., Mochón Morcillo, F. y Juan, R. D. (2012). La felicidad de los jóvenes. Papers: revista de sociología, 97(2), 407-430.

Alarcón, R. (2001). Relaciones entre felicidad, género, edad y estado conyugal. Revista de Psicología, 19(1), 27-46.

Alarcón, R. y Rodríguez, T. C. (2015). Relaciones entre gratitud y felicidad en estudiantes universitarios de Lima metropolitana. Psychologia, 9(1), 59-69.

Angelucci, L., Juárez, J. F., Dakduk, S., Lezama, J., Moreno, A. y Serrano, A. (2008). Jerarquía de valores en estudiantes universitarios. Argos, 25(48), 06-20.

Argyle, M., y Lu, L. (1990). The happiness of extraverts. Personality and individual differences, 11(10), 1011-1017.

Argyle, M., Martin, M. y Crossland, J. (1989). Happiness as a function of personality and social encounters. In J. P. Forgas, y J. M. Innes (Eds.). Recent advances in social psychology: An international perspective (pp. 189-203). North-Holland: Elsevier.

Bilbao, M. Á., Techio, E. M. y Páez, D. (2007). Felicidad, cultura y valores personales: estado de la cuestión y síntesis meta-analítica. Revista de Psicología (Lima), 25(2), 135-276.

Bolte, A., Goschke, T. y Kuhl, J. (2003). Emotion and intuition: Effects of positive and negative mood on implicit judgments of semantic coherence. Psychological Science, 14(5), 416-421. http://dx.doi.org/10.1111/1467-9280.01456

Caballero-García, P.A., Carretero Cenjor, M.J. y Fernández Palop, P. (2015). Emotional development, creativity and happiness in the initial training of teachers. Actas de la IX Internacional Conference of Education, Research and Innovation (INTED2015). Madrid, 2-4 de marzo.

Caballero-García, P.A., Carretero Cenjor, M.J. y Sánchez, S. (2016). Educación positiva: factor clave para la formación en el ámbito universitario. Comunicación presentada al XVI Congreso Nacional y VII Congreso Iberoamericano de Pedagogía. Madrid, 28-30 de junio.

Caballero-García, P.A., Carretero Cenjor, M.J., Sánchez, S. y Ruano, N. (2018). Creativity and happiness in university students. Actas de la 12th Annual International Technology, Education and Development Conference (INTED2018). Valencia, 5-7 de marzo.

Caballero-García, P.A., Ruano, N. y Sánchez, S. (2018). Happiness in university students: a descriptive study for educational improvement. Actas de la 10th annual International Conference on Education and New Learning Technologies (EDULEARN2018). Palma de Mallorca, 2-4 de julio. 
Cabrerizo Diago, J.; Rubio Roldán, M. J. y Castillo Arredondo, S. (2007). Programación por competencias. Formación y práctica. Bordón Journal of Education, 60(2), 178-179.

Carstensen, L. L. (1991). Selective theory: Social activity in life-span contex. Annual Review of Gerontology and Geriatrics, 11,195-217.

Carstensen, L. L. (1995). Evidence for life-span theory of socioemotional selective. Current Directions in Psychologilcal Science, 4(5), 151-156.

Castilla, H., Caycho, T. y Ventura-León, J. L. (2016). Diferencias de la felicidad según sexo y edad en universitarios peruanos. Actualidades en Psicología, 30(121), 25-37.

Connolly, J.J. y Viswesvaran, C. (2000). The role of affectivity in job satisfaction: A metaanalysis. Personality and individual differences, 29(2), 265-281. http://dx.doi.org/10.1016/S0191-8869(99)00192-0

Cook, T.D. y Campbell, D.T. (1979). Quasi-experimentation. Design and analysis issues for field settings. Chicago, IL: Rand McNally.

Cook, T.D. y Campbell, D.T. (1986). The causal assumptions of quasiexperimental practice. Synthese, 68, 141-180.

Cova Solar, F., Alvial, W., Aro, M., Bonifetti, A., Hernández, M. y Rodríguez, C. (2007). Problemas de Salud Mental en Estudiantes de la Universidad de Concepción. Terapia psicologica, 25(2), 105-112.

Cropanzano, R. y Wright, T.A. (1999). A 5-year study of change in the relationship between well-being and job performance, Consulting Psychology Journal: Practice and Research, 51(4), 252-265.

De la Torre, S. y Tejada Fernández, J. (2007). Estilos de vida y aprendizaje universitario. Revista Iberoamericana de Educación (OEI), 44, 101-131.

Díaz, D., Rodríguez-Carvajal, R., Blanco, A., Moreno-Jiménez, B., Gallardo, I., Valle, C. y Van Dierendonck, D. (2006). Adaptación española de las escalas de bienestar psicológico de Ryff. Psicothema, 18(3), 572-577.

Diener, E. (2000). Subjective well-being: Thescience of happiness and a proposal for a national index. American Psychologist, 55, $34-43$.

Diener, E. y Biswas-Diener, R. (2002). Will money increase subjective well-being? Social indicators research, 57(2), 119-169.

Diener, E. y Suh, M. E. (1998). Subjetive well-being and age: An international analysis. En S. K. Wamer y L. M. Powell (Eds.), Annual review of gerontology and geriatric (pp. 304324). Nueva York: Spinger Publishing.

Diener, E., Nickerson, C., Lucas, R.E. y Sandvik, E. (2002). Dispositional affect and job outcomes. Social Indicators Research, 59(3), 229-259.

Elisondo, R. C., Donolo, D. S. y Rinaudo, M. C. (2009). Ocasiones para la creatividad en contextos de educación superior. Revista de docencia Universitaria, 4, 2-16.

Fernández-Berrocal, P. y Extremera Pacheco, N. (2009). La Inteligencia Emocional y el estudio de la felicidad. Revista Interuniversitaria de Formación del Profesorado, 23(3), 85-108.

Figueiredo-Ferraz, H., Cardona, S. y Gil-Monte, P. (2009). Desgaste psíquico y problemas de salud en estudiantes de psicología. Psicologia em Estudo, 14(2), 349-35 
Francis, L. J., y Katz, Y. J. (2000). Internal consistency reliability and validity of the Hebrew translation of the Oxford Happiness Inventory. Psychological Reports, 87(1), $193-196$.

Fredrickson, B.L. y Branigan, C. (2005). Positive emotions broaden the scope of attention and thought-action repertoires, Cognition \& Emotion, 19(3), 313- 332.

Fredrickson, B.L. y Joiner, T. (2002). Positive emotions trigger upward spirals toward emotional well-being, Psychological science, 13(2), 172-175.

Frijters, P. y Beatton, T. (2012). The mystery of the U-shaped relationship between happiness and age. Journal of Economic Behavior \& Organization, 82(2-3), 525-542.

Frisch, M.B., Clark, M.P., Rouse, S.V., Rudd, M.D., Paweleck, J.K., Greenstone, A. y Kopplin, D.A. (2005). Predictive and treatment validity of life satisfaction and the quality of life inventory, Assessment, 12(1), 66-78.

Furnham, A. y Cheng, H. (2000). Perceived parental behaviour, self-esteem and happiness. Social Psychiatry and Psychiatric Epidemiology, 35(10), 463-470.

Galiana Lapera, D. R. (2015). Análisis de la felicidad, resiliencia y optimismo como factores emocionales en la inserción laboral de los universitarios (Tesis Doctoral). Alicante, Elche: Universidad Miguel Hernández.

García, M. (2002). El bienestar subjetivo. Escritos de Psicología, 6, 18-39.

García Mazzieri, S. (2012). Cultura, bienestar psicosocial y salud mental percibida (Tesis doctoral). Buenos Aires, Argentina: Facultad de Ciencias Sociales, Universidad de Palermo.

González González, C. (2014). Estrategias para trabajar la creatividad en la Educación Superior: pensamiento de diseño, aprendizaje basado en juegos y en proyectos. Revista de Educación a Distancia, 40, 1-15.

González, M., Castro, P y Martín, M. (2011). Personalidad Eficaz en Estudiantes Chilenos de Ingeniería de Primer Año. Formación Universitaria, 4(5), 3-12.

Graen, G.B. (1976). Role-making processes within complex organizations Handbook of industrial and organizational psychology (pp. 1201-1245). Chicago: Rand McNally.

Headey, B. (2010). The set point theory of well-being has serious flaws: on the eve of a scientific revolution? Social Indicators Research, 97(1), 7-21.

Heikkila, A., Lonka, K., Niemen, J. y Niemivirita, D. (2012). Relations between Teacher Students' Approaches to Learning, Cognitive and Attributional Strategies, WellBeing, and Study Success. Higher Education, 64(4), 455-471.

Helliwell, J.F. y Putnam, R. (2004). The social context of well-being. The Royal Society, 14351446.

Hernández Moreno, F. P. y Landero Hernández, R. (2014). Propiedades psicométricas de la escala de felicidad subjetiva (SHS) y su relación con el estrés, la salud percibida y el apoyo social en pacientes con cáncer de mama. Psicooncología, 11(2/3), 357.

Hills, P. y Argyle, M. (2002). The Oxford Happiness Questionnaire: a compact scale for the measurement of psychological well-being. Personality and Individual Differences, 33, 1073-1082.

Isen, A. M., Daubman, K. A., y Nowicki, G. P. (1987). Positive affect facilitates creative problem solving. Journal of Personality and Social Psychology, 52(6), 1122-1131. doi: 10.1037/0022-3514.52.6.1122 
Joseph, S. y Lewis, C. A. (1998). The Depression-Happiness Scale: reliability and validity of a bipolar self-report scale. Journal of Clinical Psychology, 54(4), 537-544.

Kashdan, T.B. y Roberts, J.E. (2004). Trait and state curiosity in the genesis of intimacy: Differentiation from related constructs. Journal of Social and Clinical Psychology, 23(6), 792-816. Recuperado de: https://doi.org/10.1521/jscp.23.6.792.54800

Labouvie-Vief, G. y Blanchard-Fields, F. (1982). Cognitive ageing and psychological growth. Ageing and Society, 2(2), 183-209. doi: 10.1017/ S0144686X00009429

Laca Arocena, F. A., Verdugo Lucero, J. C. y Guzmán Muñiz, J. (2005). Satisfacción con la vida de algunos colectivos mexicanos: una discusión sobre la psicología del bienestar subjetivo. Enseñanza e investigación en Psicología, 10(2). 325- 336

Lewis, C. A., Francis, L. J. y Ziebertz, H. G. (2002). The internal consistency reliability and construct validity of the German translation of the Oxford Happiness Inventory. North American Journal of Psychology, 4, 211-220.

Liaghatdar, M. J., Jafari, E., Abedi, M. R. y Samiee, F. (2008). Reliability and validity of the Oxford Happiness Inventory among university students in Iran. The Spanish Journal of Psychology, 11(1), 310-313.

Lu, L. (1996). Correlates of happiness among the chinese people. Taipei, Taiwan, ROC: National Science Council.

Lu, L. y Shih, J. B. (1997). Personality and happiness: Is mental health a mediator? Personality and Individual Differences, 22, 249-256.

Lyubomirsky, S (2008). La ciencia de la felicidad: un método probado para conseguir el bienestar. Barcelona: Urano.

Lyubomirsky, S. y Lepper, H. S. (1999). A measure of subjective happiness: Preliminary reliability and construct validation. Social indicators research, 46(2), 137-155.

Lyubomirsky, S., King, L y Diener, E. (2005). The Benefits of Frequent Positive Affect: Does Happiness Lead to Success? Psychological Bulletin, 131, 6, 803-855.

Medrano, M. (2014). Concepto de felicidad en jóvenes. Ajayu, 12(1), 64-78.

Mookherjee, H. N. (1997). Marital status and percepción of well-being. The Joumal of Social Psychology, 137, 95-105.

Moyano, E. y Ramos, N. (2007). Bienestar subjetivo: midiendo satisfacción vital, felicidad y salud en población chilena de la Región Maule. Universum, 22(2), 177-193.

Mroczek, D. K. y Kolarz, C. M. (1998). The effect of age on positive and negative affect: A developmental perspective on happiness. Journal Personality and Social Psychology, 75, 1333-1349.

Mroczek, D.K. y Spiro, A. (2005). Change in life satisfaction during adulthood: findings from the veterans affairs normative aging study. Journal of personality and social psychology, 88(1), 189-202.

Muratori, M., Zubieta, E., Ubillos, S., González, J. L. y Bobowik, M. (2015). Felicidad y Bienestar Psicológico: Estudio Comparativo Entre Argentina y España Happiness and Psychological Well-Being: A Comparative Study Between Argentina and Spain. PSYKHE, 24(2), 1-18.

Oliver, J. C. (2000). Multinivel regresión models: apllications in Scholl psychology. En: CSI Psicothema, 3(12), 487-494. 
Ong, A.D., Bergeman, C.S., Bisconti, T.L. y Wallace, K. (2006). Psychological resilience, positive emotions, and successful adaptation to stress in later life. Journal of personality and social psychology, 91(4), 730-749.

Ortiz, M. V., Gancedo, K. M. y Reyna, C. (2013). Propiedades psicométricas de la Escala de Felicidad Subjetiva en jóvenes y adultos de la ciudad de Córdoba-Argentina. Suma Psicológica, 20(1), 45-56.

Oswald, A. J. (1997). Happiness and economic performance. Economic Journal, 107, 18151831.

Padrós, F., Martínez, M. P., Gutiérrez-Hernández, C. y Medina, M. (2010). La psicología positiva. Una joven disciplina científica que tiene como objeto de estudio un viejo tema, la felicidad. Uaricha, Revista de Psicología, 14, 30-40.

Palomero Pescador, J. E. (2003). Breve historia de la formación psicopedagógica del profesorado universitario en España. Revista interuniversitaria de formación del profesorado, 17(2), 21-41.

Prieto Vela, A. y Muñoz-Najar Pacheco, A. O. (2015). Bienestar subjetivo e imagen corporal en estudiantes universitarias de Arequipa. Liberabit, 21(2), 321-328.

Quevedo, R. J. M., Villalobos, J. Á. G. y Abella, M. C. (2014). Relación entre bienestar subjetivo, optimismo y variables sociodemográficas en estudiantes universitarios de la Universidad de San Luis Potosí en México. Universitas Psychologica, 13(3), 10831098.

Ramírez, P. E. y Fuentes, C. A. (2013). Felicidad y Rendimiento Académico: Efecto Moderador de la Felicidad sobre Indicadores de Selección y Rendimiento Académico de Alumnos de Ingeniería Comercial. Formación Universitaria, 6(3), 21-30.

Resino, D. A. y Carrasco-Temiño, M. A. Bienestar mental y rendimiento académico en estudiantes universitarios: Un programa de psicología positiva. En J.C. Núñez, J.J. Gázquez, M.C. Pérez-Fuentes, M.M. Molero, Á. Martos, A.B. Barragán y M.M. Simón (Comp.), Psicología y Educación para la Salud (pp. 155-163). España, Oviedo: Scinfoper.

Robles Sastre, E. y Caballero García, P.A. (2010). Modelo "Campus Didáctico Profesional": Propuesta innovadora para la nueva formación del maestro en el marco del EEES. Actas del I Congreso Internacional: reinventar la profesión docente. Málaga, 8, 9 y 10 de noviembre de 2010.

Robles Sastre, E. y Caballero García, P.A. (2011). “Campus Didáctico Profesional”: Un modelo de experiencia educativa universitaria fuera del aula ordinaria y dentro del ámbito profesional. Actas del III Congreso Internacional UNIVEST: La autogestión del aprendizaje. Girona, 16 y 17 de junio de 2011.

Romero González, M. (2015). La gratitud como fortaleza humana: Una revisión bibliográfica. Tesis doctoral. Jaén: Universidad de Jaén.

Staw, B.M., Sutton, R.I. y Pelled, L.H. (1994). Employee Positive Emotion and Favorable Outcomes at the Workplace, Organization Science, 5(1), 51-71.

Ryff, C. D. (1989). Happiness is everything, or is it? Explorations on the meaning of psychological well-being. Journal of Personality and Social Psychology, 57, 1069-1081. doi:10.1037//0022-3514.57.6.1069

Salavera Borás, C. y Usán Supervía, P. (2017). Repercusión de las estrategias de afrontamiento de estrés en la felicidad de los alumnos de Secundaria. Revista 
Electrónica Interuniversitaria de Formación del Profesorado, 20(3), 65-77. DOI: http://dx.doi.org/10.6018/reifop.20.3.282601

Scorsolini-Comin, F. y dos Santos, M. A. (2012). Correlations between subjective well-being, dyadic adjustment and marital satisfaction in Brazilian married people. Spanish Journal of Psychology, 15(1), 166-177.

Staw, B.M., Sutton, R.I. y Pelled, L.H. (1994). Employee Positive Emotion and Favorable Outcomes at the Workplace, Organization Science, 5(1), 51-71.

Tomás-Sábado, J., Edo-Gual, M., Aradilla-Herrero, A., Sorribes López, J. V., Fernández-Najar, B., y Montes-Hidalgo, J. (2014). Propiedades psicométricas preliminares de la forma española del Oxford Happiness Questionnaire Short-Form (OHQ-SF) (pp. 1-7). Actas del XV Congreso Virtual de Psiquiatria. com, Interpsiquis, del 1-28 febrero.

Toribio Pérez, Lorena, González-Arratia López-Fuentes, N.I., Valdez Medina, J. L., González Escobar, S. y Van Barneveld, H.O. (2012). Validación de la Escala de Felicidad de Alarcón para adolescentes mexicanos. Psicología Iberoamericana, 20(1), 71-79.

Torres Deik, M., Moyano-Díaz, E. y Páez, D. (2014). Comportamiento juvenil universitario en busca de la felicidad: su caracterización y su eficacia. Universitas Psychologica, 13(4), 1419-1428. Recuperado de: https://doi.org/10.11144/Javeriana.UPSY13-4.cjub

Torres-Pinazo, J., Rico-Ballesteros, J., Díaz-Cabrera, J. Á., Carmona-Álamos, L., BlancoLuengo, D. y García-Tascón, M. (2017). Satisfacción laboral de los estudiantes universitarios sevillanos: Estudio piloto. El uso de datos en la Economía del Deporte. Mirando hacia el futuro, (158), 282-286.

Valiant, G. L. (1993). Life events, happiness and depression: The half empty cup. Personality and Individual differences, 15, 447-453.

Vargas, G. M. G. (2007). Factores asociados al rendimiento académico en estudiantes universitarios, una reflexión desde la calidad de la educación superior pública. Educación, 31(1), 43-63.

Velásquez C., C., Montgomery U., W., Montero L., V., Pomalaya V., R., Dioses, A., Velásquez, N., Araki, R. y Reynoso, D. (2008). Bienestar psicológico, asertividad y rendimiento académico en estudiantes universitarios. Revista de Investigación en Psicología, 11(2), 139-152.

Veloso-Besio, C., Cuadra-Peralta, A., Antezana-Saguez, I., Avendaño-Robledo, R., \& FuentesSoto, L. (2013). Relación entre inteligencia emocional, satisfacción vital, felicidad subjetiva y resiliencia en funcionarios de educación especial. Estudios pedagógicos (Valdivia), 39(2), 355-366.

Vera-Noriega, J. A. y Rodríguez, E. J. (2007). La felicidad y sus correlatos en los estudiantes de la Universidad de Sonora. Revista de la Universidad de Sonora, 19, 17-19.

Vera-Villarroel, P., Celis-Atenas, K. y Córdova-Rubio, N. (2011). Evaluación de la Felicidad: Análisis Psicométrico de la Escala de Felicidad Subjetiva en Población Chilena. Terapia Psicológica, 29(1), 127-133.

Wintre, M. G., Dilouya, B., Pancer, S. M., Pratt, M. W., Birnie - Lefcovitch, S., Polivy, J y Adams, G. (2001). Academic Achievement in First-Year University: Who Maintains their High School Average? Higher Education, 62, 4, 467-481. 
Wood, W., Rhodes, N. y Whelan, M. (1989). Sex differences in positive well-being: A consideration of emotional style and marital status. Psychological Bulletin, 106, 249264 .

Wright, T.A. y Cropanzano, R. (2000). Psychological well-being and job satisfaction as predictors of job performance. Journal of occupational health psychology, 5(1), 84-94.

Wright, T.A. y Staw, B.M. (1999). Affect and favorable work outcomes: two longitudinal tests of the happy-productive worker thesis. Journal of Organizational Behavior, 20(1), $1-23$.

Zubieta, E. M. y Delfino, G. I. (2010). Satisfacción con la vida, bienestar psicológico y bienestar social en estudiantes universitarios de Buenos Aires. Anuario de Investigaciones, 17, 277-283. Facultad de Psicología, Universidad de Buenos Aires.

Zubieta, E. M., Muratori, M. y Mele, S. (2012). Bienestar, clima emocional, percepción de problemas sociales y confianza. Anuario de Investigaciones, 19, 97-106.

Zubieta, E., Fernández, O. y Sosa, F. (2012). Bienestar, valores y variables asociadas. Boletín de Psicología, 106, 7-27.

Zubieta, E., Muratori, M. y Fernández, O. (2012). Bienestar subjetivo y psicosocial: explorando diferencias de género. Salud \& Sociedad, 3, 66-76.

Zubieta, E; Beramendi, M; Sosa, F. y Torres, A. (2010). Sexismo ambivalente, estereotipos y valores en el ámbito militar. Revista de Psicología, 29(1), 101-130. Perú: Universidad Católica Pontificia. 